\title{
Classical Cooperative Theory II: Value-Like Concepts
}

Sergiu Hart ${ }^{1}$

The Hebrew University of Jerusalem

\section{Introduction}

The Value is a solution concept originally due to Shapley (1953). The idea behind the concept is to evaluate how much will a player be willing to pay to participate in a given game. It seeks to represent what the game is worth for a player. In some sense the value captures the expected outcome of the game. We will start with the TU (transferable utility) framework and the axiomatic approach and then consider various extensions to the NTU (non transferable utility) case.

\section{TU Games}

A TU game $(N, v)$ is defined by associating a real number $v(S)$ to every coalition $S \subset N$ (put $v(\varnothing)=0$ ). $v(S)$ is referred to as the worth of the coalition $S$, i.e., what the members of the coalition $S$ can divide between them. The value will associate one p.v. (payoff vector) with each game; i.e., the value of a game will be denoted $\varphi(N, v) \in \mathfrak{R}^{N}$ where $\varphi^{i}(N, v)$ is the value of player $i$ in the game $(N, v)$.

Shapley (1953) started out with the following set of axioms.

EFF (Efficiency) (also called PO-Pareto optimality):

$\varphi(N, v)$ satisfies $\quad \sum_{i \in N} \varphi^{i}(N, v)=v(N)$.

ET (Equal Treatment) (usually called symmetry):

Two players $i, j \in N$ are called substitutes in $(N, v)$ if $v(S \cup\{i\})=v(S \cup\{j\})$ for all coalitions $S$ such that $i, j \notin S$. The axiom states that if $i, j$ are substitutes in the game $(N, v)$ then $\varphi^{i}(N, v)=\varphi^{j}(N, v)$. 
$N P$ (Null Player) (or Dummy):

A player $i$ is a null player in $(N, v)$ if $v(S \cup\{i\})=v(S)$ for all $S$. The axiom states that if $i$ is a null player in $(N, v)$ then $^{2} \varphi^{i}(N, v)=0$.

$A D D$ (Additivity):

For all players $i$ in $N, \varphi^{i}(N, v)+\varphi^{i}(N, w)=\varphi^{i}(N, v+w)$, where the game $(N, v+w)$ is defined by $(v+w)(S)=v(S)+w(S)$ for all coalitions ${ }^{3} S$.

Theorem (Shapley (1953)):

a) There exists a unique $\varphi$ satisfying EFF, ET, NP, ADD on the class of all TU games.

b) $\varphi$ is given by $\varphi^{i}(N, v)=E_{\{S \mid i \notin S\}}[v(S \cup\{i\})-v(S)]$.

The formula of $\varphi$ given in b) can be explained as follows. We look at $v(S \cup\{i\})-v(S)$ which is the marginal contribution of player $i$ to the coalition $S$, and average the marginal contributions according to a distribution over $S$. This distribution can be defined by looking at the players in a random order, where the players preceding player $i$ in a given order form the coalition $S$. One may think of the players as entering a room one by one in a random order, and averaging the contribution of player $i$, when he enters the room, to the players already in the room.

There is some distant indication of the idea of marginality in the NP (Null Player) axiom. There, a player which always contributes marginally zero gets zero value, but this is still very far from the marginal contributions that appear in the formula. It is easy to see that the four axioms are satisfied by $\varphi^{i}(N, v)=E\{S \mid \notin S\}[v(S \cup\{i\})-v(S)]$ (to see that EFF is satisfied notice that if we take the marginal contributions of all players for a given order the terms in the summation cancel each other out and we are left with $v(N)$ which is averaged over all orders). We now sketch the proof in the other direction, i.e., that the axioms imply the Shapley value. Consider a unanimity game $u_{T}$ for a given set of players $T$, i.e., $u_{T}$ is defined so that all coalitions $S$ that contain $T$ have $u_{T}(S)=1$ and all the other coalitions get 0 . By NP, players outside $T$ must get 0 , and by EFF and ET all players in $T$ must get $1 / \# T$. These unanimity games form a basis of the linear space of games. Thus by ${ }^{4} \mathrm{ADD}$ we get that the solution has to be the Shapley value since both are linear and agree on the basis of unanimity games.

As was mentioned above, the underlying notion here is "marginality", i.e., the value of a player is only a function of his marginal contributions. Thus we introduce a new axiom.

2 In the original paper by Shapley this axiom was combined with the EFF axiom.

3 Sometimes a different version of this axiom is used. It corresponds to playing the average game, and it requires that $1 / 2 \varphi^{i}(N, v)+1 / 2 \varphi^{i}(N, w)=\varphi^{i}(N,(\nu+w) / 2)$.

${ }^{4}$ Note that the value of $c u_{T}$ is $c$ times the value $u_{T}$ for all constants $c$, again by NP, $\mathrm{EFF}$ and $\mathrm{E} \Gamma$. 
MARG (Marginality):

Let $(N, v)$ and $(N, w)$ be two games (with the same set of players $N)$. Let $i \in N$. If $v(S \cup\{i\})-v(S)=w(S \cup\{i\})-w(S)$ for all $S$ such that $i \notin S$, then $\varphi^{i}(N, v)=\varphi^{i}(N, w)$.

It turns out that $\mathrm{ADD}$ which is considered as a strong axiom and NP can be replaced by MARG.

Theorem (Young (1985)):

The Shapley value is the unique solution concept satisfying EFF, ET, MARG.

One can obtain the Shapley value through yet another approach. Consider what player $j$ contributes to the value of player $i$, i.e., by how much the value of $i$ changes when $j$ "drops out": $\varphi^{i}(N, v)-\varphi^{l}(M\{j\}, v)$, (in the second term we consider the subgame of $v$ with player set $M\{j\})$. It turns out that the Shapley value satisfies that $j^{\prime}$ th contribution to $i$ 'th value always equals $i$ 'th contribution to $j^{\prime}$ th value, i.e., $\varphi^{i}(N, v)-\varphi^{i}(M\{j\}, v)=\varphi^{j}(N, v)-\varphi^{j}(M\{i\}, v)$. Moreover these equations for all $i_{2} j$ together with EFF characterize uniquely the Shapley value (Myerson (1980) and Hart and Mas-Collel (1989)). The marginal contributions above have a structural resemblance to derivatives and the requirement of equal contributions reminds us of the mixed derivatives condition of Frobenius. Taking this line of thought even further implies the existence of a "potential function" whose "gradient vector" is the value p.v. . One defines a real valued function $P$ on games as a potential if it satisfies $\sum_{i \in N}[P(N, v)-P(M\{i\}, v)]=v(N)$ for all games $(N, v)$.

Theorem (Hart and Mas-Collel (1989))

There exists a unique function $P$ satisfying $\sum_{i \in N}[P(N, v)-P(M\{i\}, v)]=v(N)$; moreover, its "derivative" is the Shapley value, i.e., $\varphi^{i}(N, v)=P(N, v)-P(M\{i\}, v)$.

In the first part of this lecture where we discussed core-like concepts the consistency axiom was shown to play a major role in axiomatization. Using a related definition of consistency yields another characterization of the Shapley value.

Theorem (Hart and Mas-Colell (1989))

EFF, ET, INV for ${ }^{5} 2$ players games and CONS characterize the Shapley value ${ }^{6}$.

Here CONS is defined as follows: let $\sigma$ be a one point solution concept. Let $(N, v)$ be a game and $T$ a subcoalition of $N$, we define the game $\left(T, v^{*}\right)$ by

$5 \mathrm{INV}$ is the axiom of covariance with respect to linear transformation (see part 1 of the lecture).

6 Notice the similarity between this characterization and Sobolev's characterization of the Nucleolus; the difference hinges on the definition of CONS. 
$v^{*}(S)=v\left(S \cup T^{c}\right)-\sigma\left(S \cup T^{c}, v\right)\left(T^{c}\right)$. Here we assume that all the players in $T^{c}$ agree that $\sigma$ is a good solution concept. We look at the subgame $\left(S \cup T^{C}, v\right)$ and subtract what the solution gives to $T^{c}$ from what the grand coalition in this game gets. The axiom states that the solution of the reduced game $\left(T, v^{*}\right)$ is the restriction to $T$ of the solution to the game $(N, v)$.

Unlike the previous definition of CONS, we use here the solution concept itself - for subgames - to define the reduced game. It turns out that in cost allocation problems this form of reduced games has a more natural appeal and indeed the Shapley value is used in such problems. The notion of value has been also extensively applied to voting games (weighted majority games). In such games the core is often empty and there may be many stable sets (some are hard to find). Clearly, the total number of votes a coalition has is usually different from its value. In these games the Shapley value (known as the Shapley-Shubik index) is best viewed as the probability that a player is pivotal. For example, if we have one big party and many small parties, the value of the large party is higher than its share of votes. But with two large parties and many small ones the power of the large parties is greatly diminished and is lower than their actual share of the votes. These phenomena implied by the value are frequently observed in practice. The value is easy to apply and is very tractable, thus it becomes a most applied solution concept. Note that the value gives us a kind of an expected outcome. Furthermore the value is linear unlike the core and the nucleolus which are only piecewise linear.

\section{NTU Games}

An NTU game $(N, V)$ is defined by associating a set $V(S) \in \mathfrak{R}^{S}$ for every coalition $S$. Consider the following diagram of classes of games (see previous chapter).

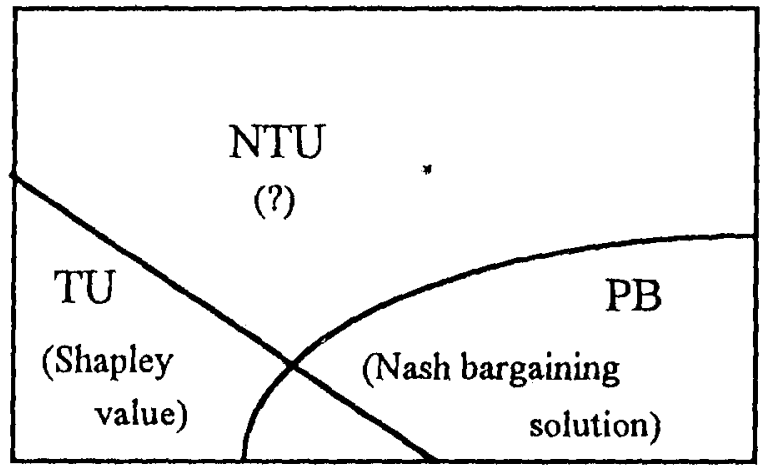

If we take the classical solutions: the Shapley value in the TU-case, and the Nash bargaining solution in the PB-case, one would like to extend these solutions to the whole space of NTU games. 
The Nash solution to 2-person PB games is characterized by the "equal angles" property, i.e., the marginal rate of substitution between the players' utilities at the solution point is equal to the ratio of the payoffs. This is shown in the following diagram.

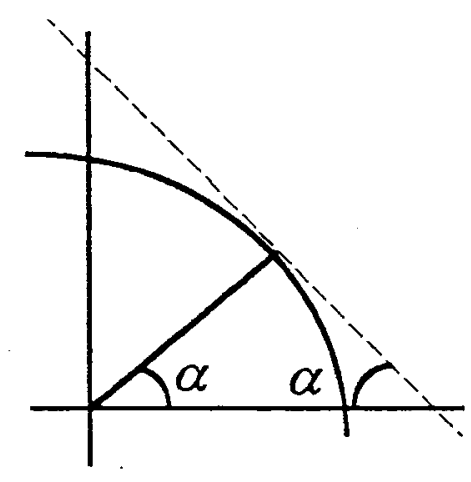

There are two underlying criteria that appear here. Let $c$ be a positive number. The first is the Utilitarian "local efficiency" criterion which means that we are on the Pareto frontier and the marginal rates of substitution there are precisely $c$ The second is the Egalitarian criterion which requires the payoffs to be distributed according to the ratio given by $c$. These two criteria are jointly satisfied at the Nash solution: i.e., they are satisfied for the same $c$. We would like to extend these criteria to the general NTU case. To do so, the first step is to define an egalitarian solution relative to a vector $\lambda$ of utility comparison weights (where $\lambda$ is a strictly positive vector in $\mathfrak{R}^{N}$ ). This solution is called the $\lambda$ egalitarian solution. It tries to capture the idea that the gains from cooperation are split equally among the players (hence comparison weights are needed). The second step consists in endogenizing the determination of the comparison weights $\lambda$. This is done by demanding that $\lambda$ be such that the $\lambda$-egalitarian solution be also $\lambda$-utilitarian, i.e., that it maximizes the sum of $\lambda$-rescaled payoffs. A fixed point theorem asserts that there are weights which will yield such a value. This approach is due to Harsanyi (1963).

A different approach was given by Shapley (1969). He looked at the induced TU game $\left(N, v_{\lambda}\right)$, given a vector $\lambda$ of utility comparison weights: $v_{\lambda}=\operatorname{Max}\left\{\sum_{i \in S} \lambda^{i} x^{i} \mid x \in V(S)\right\}$.

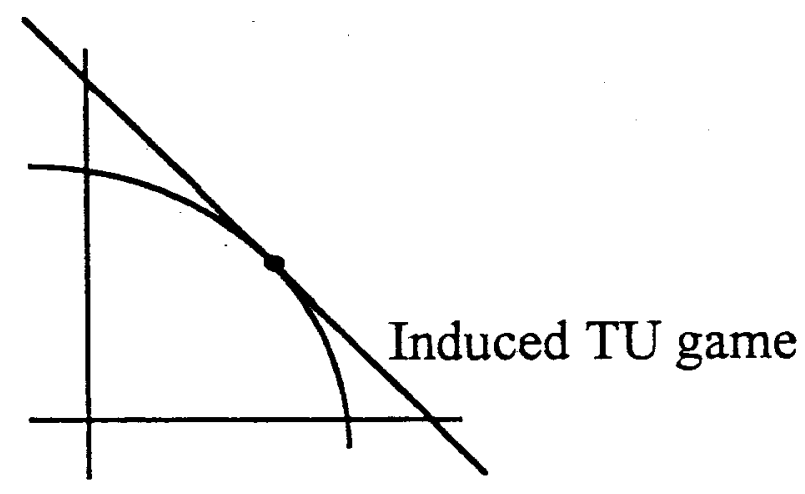


The Shapley value of the TU-induced game will be somewhere on the hyperplane and may well be non feasible in the NTU game as is shown in the diagram below.

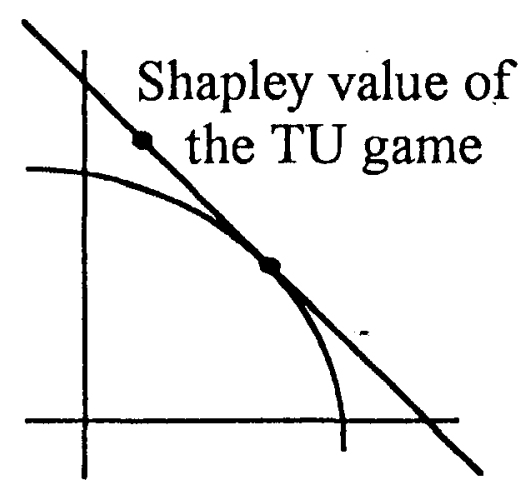

If the value of the TU game is feasible (thus the two points in the diagram above coincide) we get the Shapley NTU value. Again a fixed point argument ensures that the NTU value exists. Here we start with the marginal rate of utility substitution and check if it corresponds to the induced TU Shapley value.

There are other NTU values and some applications of values (e.g., in market games), there are also axiomatizations of the Shapley NTU value (Aumann(1985)) and of the Harsanyi NTU value (Hart(1985)).

When comparing the two approaches, i.e., Shapley's vs. Harsanyi's, it seems that Shapley's approach considers more the effect of the grand coalition on the expanse of smaller subcoalitions, whereas the Harsanyi's approach does the opposite. This can be clearly seen in their axiomatizations.

One should also mention a third NTU value. This is the consistent Maschler and Owen $(1989,1992)$ NTU value. The natural extension of CONS to NTU games is self contradicting (no solution satisfies it), so they defined an "average" reduced game. Thus they have an NTU value with a notion close in spirit to the CONS.

This entire lecture has considered the traditional approach alone. It will be seen in a later lecture how these solutions may emerge from the non cooperative approach.

\section{References}

Aumann, R.J., 1985. 'An axiomatization of the non-transferable utility value', Econometrica 53: pp.599-612..

Harsanyi, J.C., 1963. 'A simplified bargainning model for the n-person cooperative game', International Economic Review 4, pp. 194-220. 
Hart, S., 1985. 'An axiomatization of Harsanyi's non-transferable utility solution', Econometrica 53, pp. 1295-1313.

Hart, S., 1990. 'Advances in value theory', in: T. Ichiishi, A. Neyman, and Y. Tauman, eds., Game Theory and Applications. pp. 166-175. San Diego, CA. Academic Press, Inc.

Hart, S., and A. Mas-Colell, 1989. 'Potential, value, and consistency', Econometrica 57, pp. 589-614.

Maschler, M., and G. Owen, 1989. ' The consistent Shapley value for hyperplane games', International Journal of Game Theory 18, pp. 389-407.

Maschler, M., and G. Owen, 1992. ' The consistent Shapley value for games without side payments', in: Rational Interaction, R. Selten ed., Springer-Verlag, pp. 5-12.

Myerson, R. 1980. 'Conference structure and 'fair allocation rules', International Journal of Game Theory 9, pp. 169-182.

Shapley, L.S., 1953. 'A value for n-person games', in: Contributions to the Theory of Games II (Annals of Mathematics Studies 28), H. Kuhn and A. Tucker, eds., Princeton, NJ: Princeton University Press. pp. 307-317.

Shapley, L.S., 1969. 'Utility comparison and the theory of games', in: La Decision. Paris: Editions du CNRS, pp. 251-263.

Young, H.P., 1985. 'Monotonic solutions of cooperative games', International Journal of Game Theory 14: pp. 65-72. 